\title{
EFFECT OF LOCAL TEXTURE ON THE DEVELOPMENT OF M-K GROOVE IN BIAXIAL DEFORMATION
}

\author{
W.B.LEE and K.C.CHAN \\ Department of Manufacturing Engineering, Hong Kong \\ Polytechnic, Kowloon, HONG KONG.
}

\begin{abstract}
The postulation of a groove defect is the cornerstone of the Marciniak-Kuczynski (M-K) theory in the prediction of the biaxial limit strains of sheet metals. Without the assumption of an initial thickness inhomogeneity, such a groove is shown herein to arise from texture colonies and its size predicted based on a knowledge of the texture variation in commercial sheets.
\end{abstract}

\section{INTRODUCTION}

In biaxial stretching, plastic strain increment occurs in all directions of the sheet surface and no inextensional direction can be found. Marciniak and Kuczynski (MK) 1 have postulated the existence of an initial imperfection in the sheet which will develop into a groove running in a direction perpendicular to the larger principal stress. However, the value of the groove depth in the initial sheet which must be chosen to make the experimental limit strain in equibiaxial tension match the prediction of the theory is too large and is unlikely to exist. Various equivalent forms of the "groove" have been suggested which include surface roughness, inclusions or voids. Recently it has been shown by Lee and Chan $^{2}$ that such a groove can arise from the difference in the thickness strains among grains of different crystallographic orientations taken along different strain paths during stretching. The effect of crystallographic textures on the formability of sheet metals is well known but the effect of local textures on the limit strains is less explored. It is the aim of this paper to invesitgate the effect of local texture variation on the growth of the groove defect. 


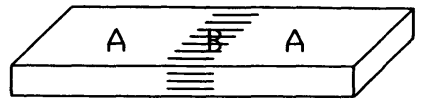

(a)

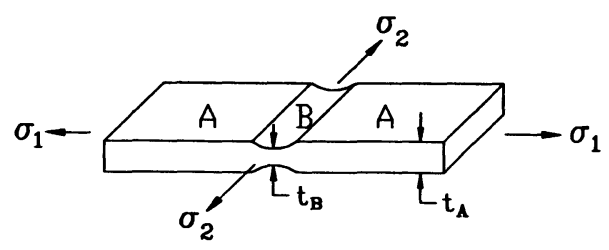

(b)

Fig. 1. Geometry of groove development.

Fig.1 shows a sheet metal with a region $B$ having a local texture different from the mean texture in region $A$. For a textured material deformed in biaxial tension, the strain path may not be linear ${ }^{3}$. Upon stretching, region $A$ and $B$ will take up different strain paths. The strain in the transverse direction is assumed to be the same in both the $A$ and $B$ region and the difference in the thickness strains will show up as a groove lying perpendicular to the rolling direction. The principal strain axes are taken as coincident with the long axis of the groove. In the region $A$, the imposed plain stress state is denoted by

$$
\sigma_{A}=\sigma_{A}\left[\begin{array}{lll}
1 & 0 & 0 \\
0 & 1 & 0 \\
0 & 0 & 0
\end{array}\right]
$$

The corresponding strain tensor of equation (1) is denoted by

$$
\epsilon_{A}=d \epsilon_{A}\left[\begin{array}{ccc}
x_{A} & 0 & 0 \\
0 & 1-x_{A} & 0 \\
0 & 0 & -1
\end{array}\right]
$$

where $x_{A}$ is a dimensionless parameter. In the groove region $B$, force equilibrium gives

$$
\sigma_{1 B}=\sigma_{1 A} / f
$$

where $f$ is the inhomgeneity factor and equals $\left(t_{B} / t_{A}\right)$. The groove strain $d \epsilon_{2 B}$ is assumed to be the same as the corresponding strain outside the groove:

$$
d \epsilon_{2 B}=d \epsilon_{2 A}=d \epsilon_{A}\left(1-x_{A}\right)
$$


The strain tensor in the region $B$ can be written as

$$
\epsilon_{B}=d \epsilon_{B}\left[\begin{array}{ccc}
x_{B} & 0 & 0 \\
0 & 1-x_{B} & 0 \\
0 & 0 & -1
\end{array}\right]
$$

where $d \epsilon_{B}=\left(1-x_{A}\right) d \epsilon_{A} /\left(1-x_{B}\right)$

When the groove is parallel to the rolling direction (RD), equations (3), (4) and (6) will be replaced by

$$
\text { and } \begin{aligned}
\sigma_{2 B} & =\sigma_{2 A} / f \\
d \epsilon_{1 B} & =d \epsilon_{1 A}=x_{A} d \epsilon_{A} \\
d \epsilon_{B} & =x_{A} d \epsilon_{A} / x_{B}
\end{aligned}
$$

respectively. The stress components $\sigma_{i j}$ and strain components $\epsilon_{i j}$ are related by the normality principle:

$$
\epsilon_{i j}=\lambda \partial F\left(\sigma_{i j}\right) / \partial \sigma_{i j}
$$

where $F\left(\sigma_{i j}\right)$ is the yield function of the sheet metal and $\lambda$ is the porportionality constant. In this paper, the crystallographic based non-integer yield function proposed by Montheillet ${ }^{5}$ is used. To calculate $\epsilon_{A^{\prime}}$ the parameter $x_{A}$ in equation (2) is varied between 0 and 1 until the $\sigma_{i j}$ equals the component of the imposed stress tensor of equation (1). $\epsilon_{B}$ is also determined by varying $x_{B}$ in equation (5) until the force equilbrium requirement of equation (3) or (7) is met. For each small increment of deformation, the change in the crystal rotation of each grain is followed and the strain tenosr recalculated ${ }^{3}$. The inhomogeneity factor after each samll step of deformation is given by

$$
f=\exp \left(\epsilon_{3 B}\right) / \exp \left(\epsilon_{3 A}\right)
$$

The procedure of determining $f$ is repeated until $d \epsilon_{1 B} \gg$ $d \epsilon_{1 A}$ ie., the strain state in the groove region approaches plain strain. The major strain outside the groove $\epsilon_{1 \mathrm{~A}}$ will give the limit strain of the sheet in equi-biaxial tension. 


\section{RESULTS AND DISCUSSION}

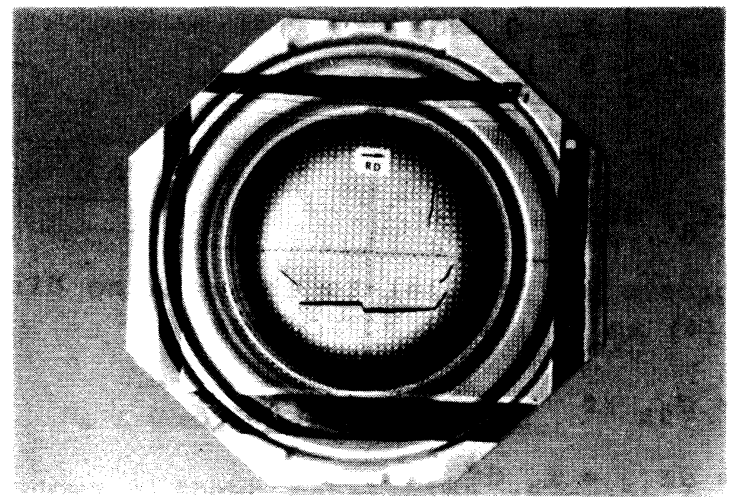

Fig. 2. Necking failure in biaxially stretched aluminium sheet.

An numerical example showing the effect of local texture variation on the magnitude of the inhomogeneity factor $f$ is illustrated for a $0.8 \mathrm{~mm}$ thick annealed commercial purity aluminium sheet. Samples of the aluminimum sheet were stretched in a double action press over an auxiliary steel bank with a central hole diameter of $30 \mathrm{~mm}$. Necking failures were frequently observed in both the rolling and transverse direction (Fig.2). The orientation distribution function of the sheet was determined by three incomplete pole figures on a Philip APD10 X-ray texture goniometer. The volume fraction of the main texture components is represented by $\{112\}<111>(548)+\{100\}<001>(27 \%)+\{123\}<634>(198)$. In the region $B$, the texture is assumed to consist of more cube components ie., $(1+x) v_{c}+\left(v_{a}-x / 2\right)+\left(v_{b}-x / 2\right)$, where $v_{c^{\prime}} v_{a}$ and $v_{b}$ is the volume fraction of the $\{110\}<001>,\{112\}<111>$ and $\{123\}<634>$ texture components in the mean textured material respectiveluy, and $x$ is the volume fraction of extra cube components in the $B$ region. Such a segregation of cube components has been reported in aluminimum sheets ${ }^{5}$.

(a) Groove $\perp \mathrm{RD}$

The simulated strain path of the region outside and within the groove is shown in Fig. 3 for $x=0.05$ and $x=0.3$. It can be seen from Fig. 3 that as the cube texture components in the groove region increases the strain paths followed by the region $A$ and $B$ diverge rapidly. The cube texture colony has a tendency to deform towards the plane strain state when $\epsilon_{2 A}$ reaches about 0.22 . The change in the homogeneity factor $f$ with thickness strains 


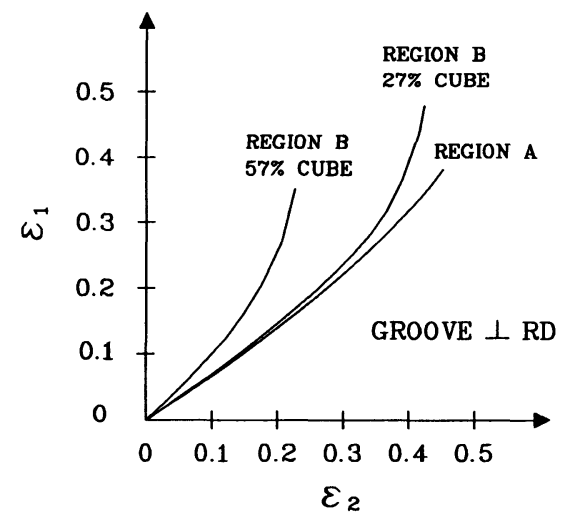

Fig. 3. Strain paths outside and within the groove.

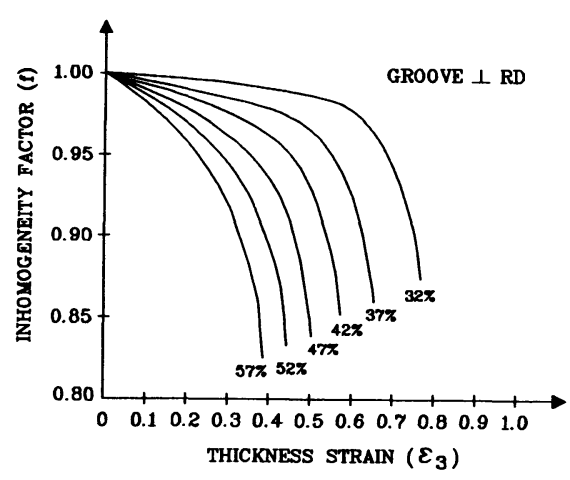

Fig. 4. Changes of inhomogeneity factor with different amounts of cube components in region $B$.

for different amounts of cube components in the groove region is shown in Fig.4. Different $\&$ of cube texture components are assumed in the groove region. The thickness rato is found to decrease rapidily with straining and the rate of decrease increases with the amount of cube components in the groove.

(2) Groove // RD

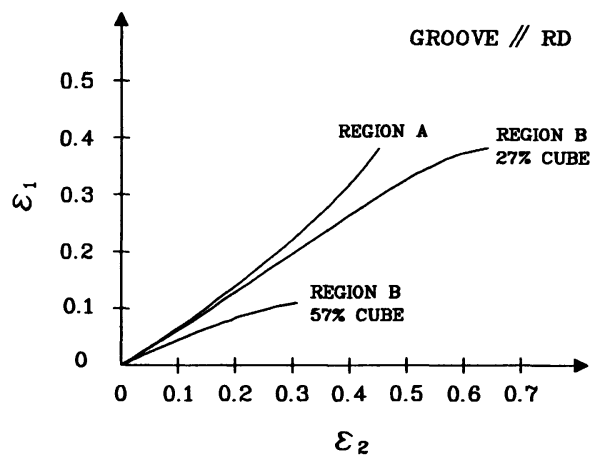

Fig. 5. Strain paths outside and within the groove.

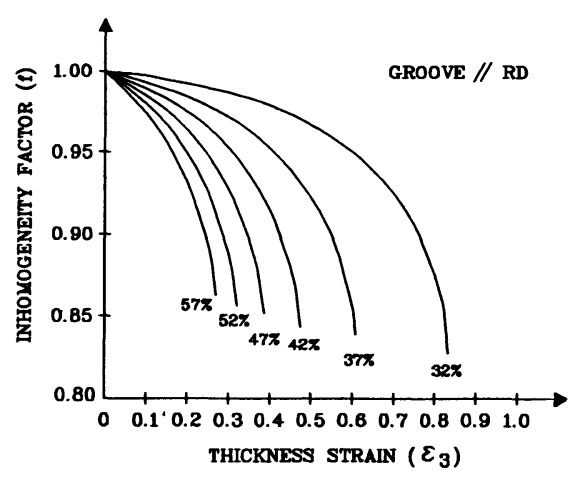

Fig. 6. Changes of inhomogeneity factor with different amounts of cube components in region $B$.

The strain path followed by the $A$ and $B$ region is shown in Fig.5. The textures used for the simulations are the same as for the previous case. When the extra cube components in the groove increase from 5\% to 30\%, the strain along the transverse direction increases more rapidily than that along the rolling 
direction. A plain strain state is almost reached in the groove as $\epsilon_{1 A}$ approaches 0.11 . The development of the inhomogeneity factor is similar to the previous case but the limit strain is reached earlier when the groove is parallel to the rolling direction.

The above analytical results shows that a $M-K$ groove of sufficient large size can be developed without assuming any initial defects or "equivalent defects" in an initial damage-free sheet. The growth of the groove is sensitive not only to the texture variations but also to the alignement of the texture colony. For a segregation of about 40 \& cube compoents in the groove region ( ie., 30 \& more than the average texture) the maximum biaxial limit strain that can' be acheived is $\left(\epsilon_{1 \mathrm{~A}}=0.109\right.$, $\epsilon_{2 A}=0.1598$ ) when the groove is parallel to the $R D$. When the groove is perpendicular to $R D$, the same amount of cube segregation will give a biaxial limit strain of $\left(\epsilon_{1 A}=0.1605\right.$, $\epsilon_{2 A}=0.2263$ ). Although the importance of the plastic anisotropies of individual grains is well known in literatures ${ }^{5}$, many theoretical predictions of the forming limits still rely on the hypothetical assumption of an initial defect size. This paper is the first attempt to predict the size of the groove from the amount of segregated texture components without recourse to the original anlysis of the $M-K$ model. Such information will be needed for the industrial control of the texture variation in sheet metals.

\section{References}

1. Z. Marciniak and K. Kuczynski, Int. J. Mech. Sci., 9, 609 (1967).

2. W.B.Lee and K.C. Chan, Proc. of the 3rd International Conference in Technology of Plasticity, Kyoto, July 1-6, p.1285, (1990).

3. K.C.Chan and W.B.Lee, Int. J. Mech. Sci, 32, 467 (1990).

4. F. Montheillet, P. Gilormini and J.J.Jonas, Acta Metall., 33, 705 ( 1985 ).

5. D.V. Wilson, W.T. Roberts and P.M.B.Rodrigues, Met. Trans. 12A, 1595 (1981). 